\title{
ENTRE ÍBEROS, CELTÍBEROS Y VASCONES. INSCRIPCIONES INÉDITAS Y REVISADAS DE TIERRAS ALTAS DE SORIA
}

\author{
AMONG IBERS, CELTIBERS AND VASCONES. UNPUBLISHED \\ AND REVISED INSCRIPTIONS FROM TIERRAS ALTAS DE SORIA
}

\author{
Eduardo Alfaro Peña \\ Proyecto Idoubeda oros \\ † Joaquín Gómez-Pantoja Fernández-Salguero \\ Universidad de Alcalá de Henares
}

\begin{abstract}
RESUMEN. Se presentan cuatro novedades epigráficas latinas localizadas en Tierras Altas de Soria, dos de ellas estelas funerarias que incluyen una onomástica indígena no indoeuropea. En segundo lugar, se plantean reflexiones que pudieran justificar la presencia de estos nombres locales en época altoimperial en un entorno tradicionalmente céltico.
\end{abstract}

PALABRAS CLAVE. Inscripción latina, estela funeraria, Tierras Altas de Soria, onomástica local.

ABSTRACT. Four new Latin inscriptions from Tierras Altas de Soria are presented; two of them are funerary stelae that include a non-Indo-European indigenous onomastics. Secondly, some considerations in order to explain the presence of these local names at Early Empire in a traditionally Celtic environment are discussed.

KEYWORDS. Latin inscription, funerary stele, Tierras Altas de Soria, local names.

* Correspondencia a / Correspondence to: Eduardo Alfaro Peña, C/ Las Peñas 18 (42176 Santa Cruz de Yanguas, Soria) - idoubeda@gmail.com https://orcid.org/0000-0002-8300-2348.

Cómo citar / How to cite: Alfaro Peña, Eduardo; † Gómez-Pantoja Fernández-Salguero, Joaquín (2021), «Entre íberos, celtíberos y vascones. Inscripciones inéditas y revisadas de Tierras Altas de Sorian, Veleia, 38, 165-182. (https://doi.org/10.1387/veleia.22079).

Recibido: 14 septiembre 2020; aceptado: 16 octubre 2020.

ISSN 0213-2095 - elSSN 2444-3565 / (C) 2021 UPV/EHU

(c) (i) () $\Theta$ Esta obra está bajo una licencia

Creative Commons Atribución-NoComercial-SinDerivadas 4.0 Internacional 
Si se tienen en cuenta los hallazgos epigráficos de las últimas cuatro décadas en Soria, estos han sido fructíferos en sus Tierras Altas, tres valles del interior del Sistema Ibérico, los del Cidacos, Linares y Alhama, que nacen en el sector castellano-leonés de la Serranía y tras encajonarse en las duras barranqueras que separan la provincia de Soria de La Rioja, se abren a las vegas del valle del Ebro al que vierten en torno a Calahorra y Alfaro.

Este aumento de inscripciones, importante por ampliar la visión tanto de su romanización a través de la onomástica latina como del mundo simbólico indígena que aparece reflejado en las decoraciones de registro inferior de las estelas, se ha complementado con el sucesivo enriquecimiento en los epígrafes de una onomástica indígena que pone en entredicho los presupuestos indoeuropeos y célticos que la tradición investigadora ha venido atribuyendo a este sector de la serranía soriana.

Hasta la publicación de la Epigrafía de la Provincia de Soria (Jimeno 1980) solo se conocían 11 inscripciones; cuarenta años después, el corpus epigráfico comarcal asciende a 391. De las 11 inscripciones citadas, 6 se correspondían con unas estelas funerarias características de la zona, que repiten una distribución sobre el soporte y unas fórmulas más o menos fijas, de ahí que podamos hablar de una officina, de un taller lapidario, eso sí, con los matices y las características propios de un territorio rural y de montańa. Hoy son ya 29 las estelas conocidas que guardan la estructura del taller, cuyo marco cronológico se ha situado entre la segunda mitad del siglo i y mediados del siglo II. Es en estas estelas altoimperiales en las que aparecen, entre unos mayoritarios nomina y cognomina latinos, los reflejos onomásticos del pasado prerromano.

Antes del trabajo de Jimeno solo una de las estelas contaba con uno de estos nombres indígenas, Lesuridantaris (gen.), que anunciaba lo que en las últimas décadas ha ido afianzándose. A mediados del siglo pasado, J. Caro Baroja (1946, 150-155) y ańos después M. a L. Albertos (1966, 130 y 274-275) ya apuntaban la proximidad al ibérico y al vasco antiguo de este nombre; Albertos argumentó esta presencia como una posible pervivencia de reductos de iberismo en el interior de la Serranía ante el avance celtibérico.

Claves serán, la década siguiente, los trabajos de U. Espinosa, especialmente el que comparte con L. M. Usero. Espinosa y Usero conocen ya 17 estelas del taller, en las que aparecen nuevos nombres indígenas como Oandissen, Arancisis (gen.) y Agirseni (gen.). Con estas novedades onomásticas aprecian vínculos de las gentes de las estelas con el mundo ibérico, señalando a Calagurris como la referencia urbana romanizadora de la que dependerían mediante adtributio (Espinosa \& Usero 1988; Espinosa 1992, 907-908).

La aparente relación altoimperial con una ciudad del Ebro del convento cesaraugustano incide en cuestionar la tradicional filiación pelendona y cluniense de estos valles sorianos del Ebro, tradición sustentada en la asociación de una cultura arqueológica, la Cultura Castreña Soriana de los siglos VI-IV a.C., con un etnónimo de las fuentes, los pelendones (Albertini 1923, 100; Taracena 1933, 395), mencionados por primera vez en el siglo i a.C., a los que Plinio (Nat. hist. IV, 112) un siglo después sitúa en el nacimiento del Duero y les atribuye también Numancia.

Las piezas localizadas en las dos últimas décadas insisten en volver la vista al mundo ibérico-vascón del valle del Ebro para desentrañar la realidad del substrato indígena en el territorio; los nuevos nombres que se van conociendo como Sesenco, Onse, Onso, Attasis (gen.), etc., hacen que se haya hablado ya de un «área antroponímicamente vascona» (Gorrochategui 2009, 543-544), aunque sin obviar la aparición de nombres célticos como Balani (gen.) (Gómez-Pantoja \& Alfaro 2001, 173-175) o el

\footnotetext{
1 Siendo rigurosos, son 35 las sorianas; las cuatro restantes proceden de la Serranía inmediata de La Rioja (Valdeosera, Munilla, San Vicente y Grávalos), repre-
}

sentando a día de hoy el ya muy difuso confín norte del modo de hacer epigráfico centrado en el sector soriano de la Sierra. 
Ambatus de una de las novedades que se presentan, así como la evidencia de deidades célticas como las Matres.

Presentamos aquí cuatro novedades y propuestas, así como algunas reflexiones a propósito de los orígenes de la onomástica indígena que caracteriza a la epigrafía de Tierras Altas. Dos de las novedades son estelas altoimperiales que mantienen las características del taller; ambas se conocían con anterioridad, aunque al estar empotradas en construcciones tradicionales su lectura era parcial, incluso, en uno de los casos, muy limitada. Los otros dos epígrafes son de cronología avanzada, ajenos en el tiempo y a los usos del taller. Uno totalmente inédito, el otro dado a conocer por T. Ortego en la década de los setenta y que revisamos (Ortego 1976, 257-258). Sirva esta mención a Ortego para reconocer su valor en la historiografía epigráfica comarcal, pues será su trabajo de campo publicado tardíamente (Ortego 1988) en el que se sustentarán las aportaciones de Espinosa.

\section{Villartoso 1 (figs. 1 y 5)}

Lastra de arenisca tallada en forma rectangular, cuyas dimensiones actuales son $(119) \times(55) \times 10 \mathrm{~cm}$. Está mutilada por la parte inferior, con una rotura diagonal que afecta al motivo decorativo bajo el texto y al final del penúltimo renglón del letrero; el lado derecho fue también recortado, y falta parte de algunas letras finales, así como la esquina superior derecha de la cabecera. Esta se adorna con dos cabezas silueteadas, de tamaño similar y pulcro trazo, que únicamente difieren en que en el lado izquierdo se grabaron los rasgos faciales (ojos y cejas, nariz y boca), mientras que el contiguo es liso y le falta la parte superior derecha del cráneo, que desapareció con la rotura de la piedra. Por debajo de los retratos, siete líneas de texto con dos epitafios: el primero, de tres líneas, se grabó mediante repicado y se caracteriza porque no hay espacio interlineal, que sí se respetó en el segundo texto, cuyas letras se marcaron someramente. Estas son capitales de trazo descuidado y con rasgos arcaizantes, como la L caudada y la E de dos trazos verticales, midiendo ca. $5 \mathrm{~cm}$. Finalmente, bajo el texto, el acostumbrado friso con animales, uno por cada difunto, pero debido a la mutilación del pie de la estela solo queda la cabeza y el lomo de un équido con el repunte de la cola.

Descubierta en 2016 al limpiar y consolidar la Fuente Ayá, situada extramuros del caserío. Servía de mampuesto en uno de los lados del arcosolio que protege el cańo y la pila de la fontana, con más de sus tres cuartas partes incrustadas en el muro, mientras que el resto sobresalía por la cara externa para servir de asiento a los usuarios y sus vasos. Extraída en 2019, está depositada en la iglesia de Villartoso.

Âemilia · Sulagessia / Ambati filia · ano(rum) ${ }^{3}$ / LXV Hic s(epulta) est / Âemililius · Udan/us Bugan.sonis / filius · an(norum) $\cdot$ C Hic s(epultus) est.

Noticia del hallazgo y transcripción de la parte visible in situ en Alfaro 2018a, 66.

Se trata de un doble entierro, grabado por manos distintas y seguramente con un intervalo de alguna década entre ambos decesos; no hay indicación de parentesco entre los difuntos, pero es probable que se trate de una pareja, de la que habría fallecido primero la mujer y luego el varón, cuya longevidad fue ciertamente excepcional. Tanto la morfología de la pieza como la disposición del letrero coinciden con los de la lápida dúplice de Antestia Onse, muerta con 25 años, mientras que quien debió de ser su cónyuge, Aemilius Seranus, lo hizo a los 50 (Gómez-Pantoja \& Alfaro 2001, 179-181; HEpOl7594). 


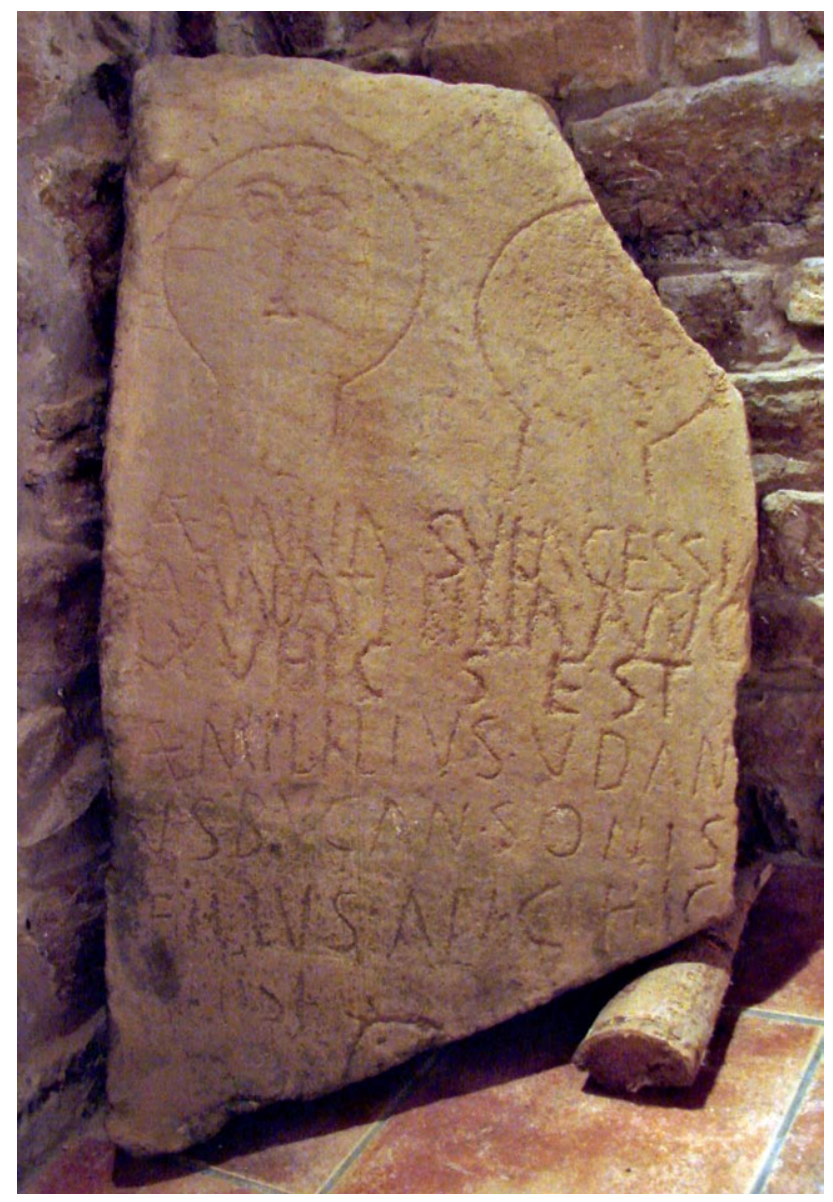

Figura 1. Villartoso 1.

Ambos compartieron el gentilicio, que es uno de los más corrientes en la comarca y cuya expresión plenis verbis no obvia el compendio de las dos primeras letras. Pero el gentilicio y el patronímico de la mujer es lo único vulgar que tiene la onomástica de ambos individuos; los restantes nombres son excepcionales y carecen de paralelos conocidos, tanto en la zona del hallazgo como en el resto de Hispania y en las demás provincias del Imperio. Udanus puede ser una variante sorda de un poco corriente nomen gentile Utanius, que usaron unos pocos individuos de Roma y sus alrededores. Los sufijos finales de Sulagessia y Bugansonis, ajenos al ámbito indoeuropeo, están atestados en Tierras Altas, en otros pocos lugares del valle del Ebro y de forma más corriente en Aquitania, y se asocian corrientemente con sustratos ibéricos o protovascónicos ${ }^{2}$.

Si fuera una inscripción procedente de las comarcas próximas más romanizadas, el nombre de los difuntos en nominativo, la parquedad de las biografías y la más que simple declaración del lu-

2 Sobre -sonis vid. los ejemplos recogidos en Gorrochategui 1984, 369, a los que deben añadirse Onso, Onsonis, respectivamente Abisunsonis (gen.) (HEp 8, 369). 
gar del entierro permitirían datar el epígrafe en el siglo i. Pero procediendo de una zona remota, esos criterios cronológicos no son necesariamente los más acertados. Lo que sí se puede afirmar por comparación con otros ejemplos del corpus local es que se trata de una de las inscripciones más tempranas por prescindir de la invocatio, por la ausencia de abreviaturas (incluso aquellas tan obvias como filius/-a), la omisión de los dedicantes y la carencia de expresiones sentimentales.

\section{Villartoso $2^{3}$ (figs. 2 y 5 )}

Estela funeraria sobre losa de arenisca, burdamente labrada y en la que no se corrigieron los defectos superficiales de la cara inscrita. Aun faltando el busto característico de estos epígrafes, parece que se conserva íntegro por arriba. También conserva su anchura original, aunque ambos costados tienen sus aristas desgastadas y en ocasiones melladas, sin que ello afecte al letrero; dimensiones actuales $122 \times 44 \times 10 \mathrm{~cm}$. La estela consta de un doble registro, siendo el inferior la representación de un cuadrúpedo en carrera. El letrero se grabó sin guías adaptándose a las deficiencias de la piedra, como sucede en las ll. 2 y 3, donde las dos últimas letras se grabaron en la amplia exfoliación de la esquina superior derecha. Se usó el repicado para grabar las letras, que son capitales de factura y tamaño irregular $(7-4 \mathrm{~cm})$, y que en algunos casos mezclan aleatoriamente formas cursivas con las ordinarias (E) o invierten su trazo $(\mathrm{S})$.

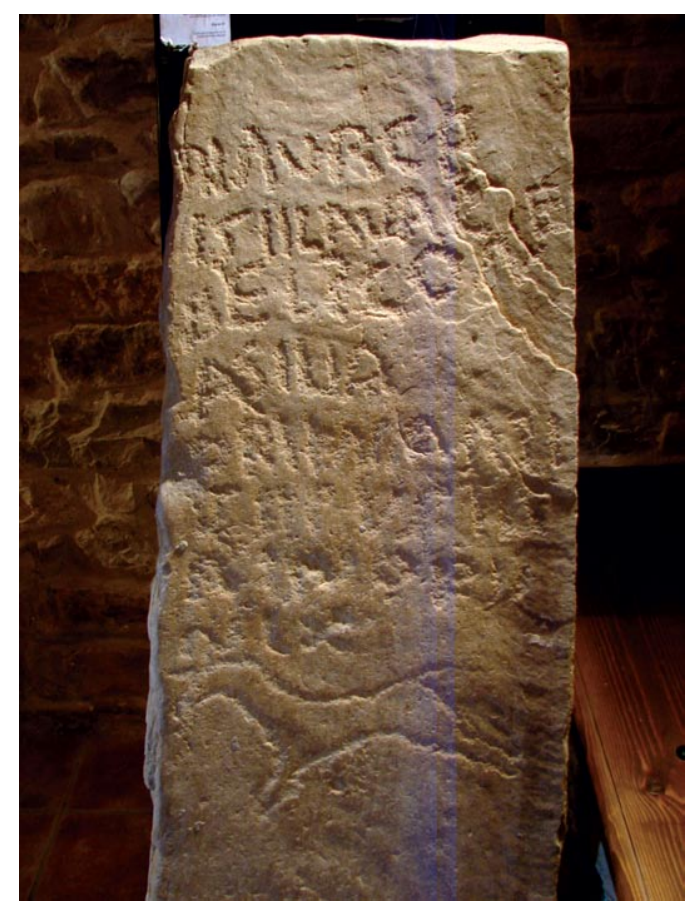

Figura 2. Villartoso 2.

3 Agradecemos las apreciaciones de J. Gorrochategui en gratificante visita realizada el 17 de enero de 2020 en compañía de su mujer, que nos han permitido clarificar algunos puntos de esta complicada pieza. 
Con su cara inscrita a la vista, sirvió de umbral en la entrada de una casa de donde fue extraída en el verano de 2016 para depositarla en la iglesia del pueblo.

Se trata de un epitafio individual, como se desprende de la única mención de la edad y la solitaria imagen de un animal, sin indicios claros de sexo o especie, aunque puede ser de cérvido. La pésima conservación del texto por la erosión de la superficie de la piedra, la imprecisa grafía de las letras y la ausencia de interpunción dificultan enormemente la lectura, que ha sido finalmente posible gracias a las autopsias realizadas y a los modelos digitales obtenidos del levantamiento fotogramétrico realizado en 2019. El resultado es un epitafio que se adhiere al poco imaginativo formulario de los epígrafes comarcales, donde lo más singular es la onomástica de la difunta y su pariente, porque mezcla los nombres personales más vulgares con otros sorprendentes y para los que no suele haber paralelos. La lectura resultante es vulgar en su propósito y formulario: una hija sepulta a su madre, pero los nombres de ambas tienen su punto de incertidumbre.

$$
\text { +AVRCE / ISIILAVRC E 3/ BELSCON / Asilia / +++ ma'ț̣i \% sepu' l'ta / annoru/m LX }
$$

Hallazgo y transcripción de la parte visible in situ en Alfaro 2014, 325 y 2018b, 339.

1. La cruz puede corresponder a una letra indistinta, tal vez $\mathrm{H}$ o $\mathrm{L}$, a la que sigue una A sin filete. AVRCE carece de paralelo, pero se asemeja a Laurco, documentado entre los Auscii aquitanos: Laurco Montanusf. (CIL XIII 472).

2. ISIILAVRC E, lapis. La serie carece de paralelo conocido y tampoco se ajusta a lo habitual en la nomenclatura latina o hispana. Dada la semejanza de la secuencia final con el renglón anterior, la separación de palabras es obligada: Ise l Aurce; de nuevo el primer nombre carece

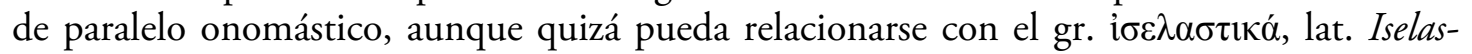
tica.

3. La raíz -belsco consta en el epitafio de Lutbelscottius, hallado en San Román de San Millán, cerca de Vitoria.

4. Se trata de un infrecuente nombre personal y sorprende el vacat que le sigue a la vista de la corta longitud de la palabra siguiente. Hay al menos un nombre propio latino que es un estricto paralelo de Sigus, en Numidia .

5. ORII, lapis. Posiblemente Hora (gr. " $\Omega \rho \alpha$ ), con omisión de la consonante muda. MARII, lapis.

6. SEPVSTA, lapis, con la segunda $S$ trazada de forma refleja, rasgo atestiguado en una lápida de San Pedro Manrique.

7. AINORV, lapis.

8. El trazado de la $\mathrm{M}$ es peculiar.

\section{Santa Cecilia 1 (figs. 3 y 5)}

Laja de arenisca empleada tal y cual fue extraída, como muestra la ausencia de labra en los costados y el acomodo del texto a las imperfecciones de la piedra. La cara inscrita ha perdido parte del borde derecho y una extensa exfoliación afectó al comienzo de los renglones tercero a quinto, bo-

${ }^{4}$ CIL VIII 19149; Gsell \& Pfaum 1976, cat. n. ${ }^{\circ} 6551$. 
rrando parcialmente el texto. Sus dimensiones actuales son $(73) \times(35) \times 10 \mathrm{~cm}$. El texto se reparte en 11 líneas, burdamente incisas sin ayuda de interlineado, lo que resulta en renglones torcidos, interlineado irregular y letras capitales incisas de modo descuidado y cuyo tamańo disminuye a medida que avanza el epitafio, de tal modo que el primer renglón, resaltado por dos líneas incisas horizontales, mide $4 \mathrm{~cm}$ y las del último, $2,2 \mathrm{~cm}$. Nótese que algunas letras presentan formas cursivas con otras regulares; eso sucede con la R, cuyo trazo cursivo puede confundirse fácilmente con la $\mathrm{A}$, que se representa sin brazo; la $S$ combina formas con las adecuadas curvas con otras más aplanadas.

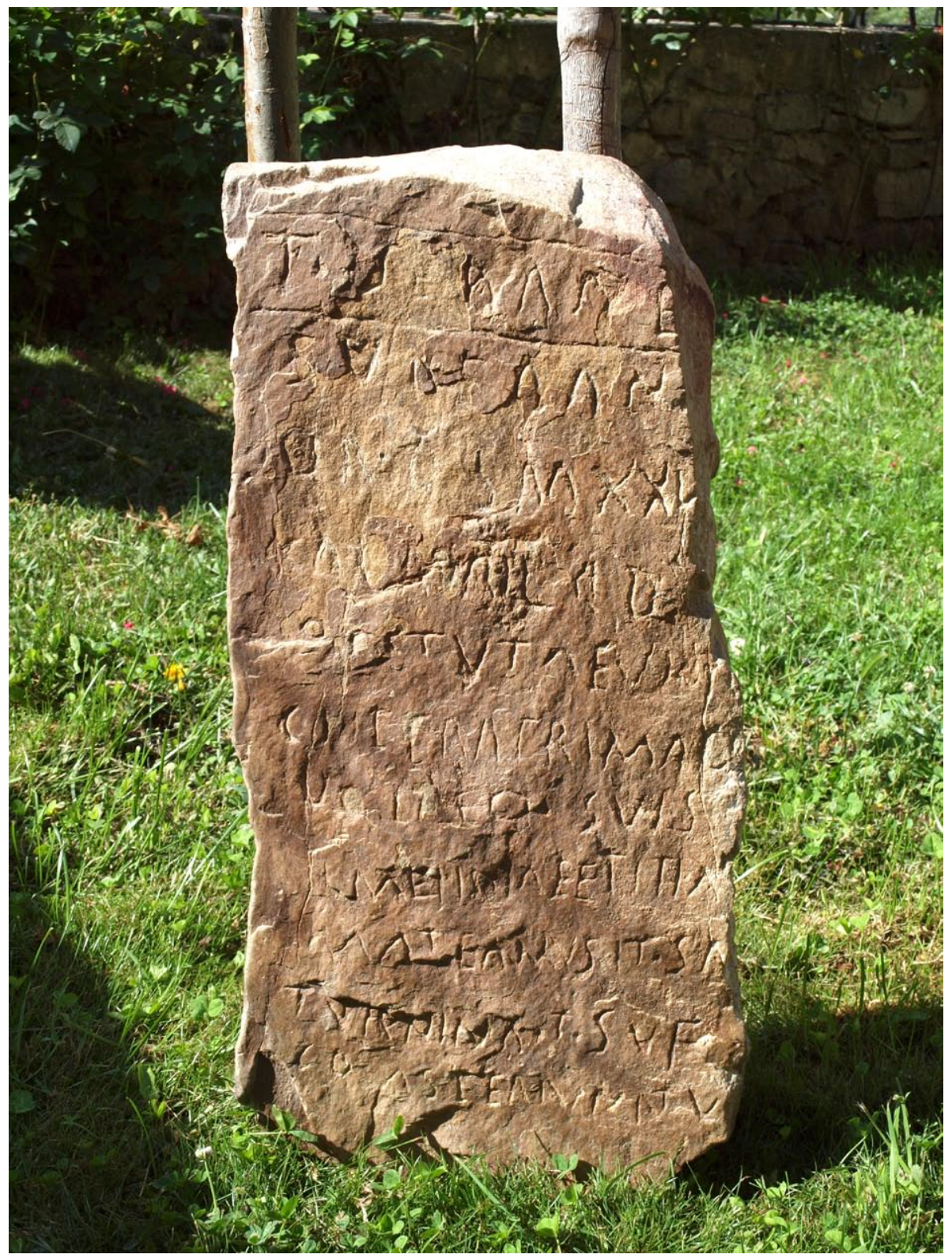

Figura 3. Santa Cecilia 1. 
Encontrada en torno a 2006 durante la reforma de una casa de Santa Cecilia, permanece exenta en el interior del edificio en que se encontró 5 .

La lectura que sigue, dificultosa por las características del epígrafe, se ha hecho a partir de varias autopsias y fotografías realizadas desde su descubrimiento hasta el verano de 2019, cuando se llevó a cabo el levantamiento fotogramétrico.

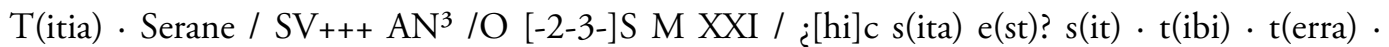
l(evis) + D / +++tutae VN6 / CS+T fratri MAO / VOTAEO • suis / Melidia et Titi(us) • 9/ Maternus et $\mathrm{Sa} /$ turnina $\cdot \mathrm{t}($ itulum $) \cdot \mathrm{su}(\mathrm{is}) \mathrm{f}$ (ecerunt) / CO Asteriu ânn(is) V

1. T(itia), a partir de la aparición de este nombre en v. 8. Serane: aunque en el grupo hay dos ejemplos de un mismo nombre femenino que hace el nominativo singular en -e, Pontia Onse Onsonis f. de Yanguas y Antestia Onse Murrani f. de Navabellida (Gómez-Pantoja \& Alfaro 2001, 179181; HEpOl 15205 y 7594), la norma es el respeto a la morfología canónica, salvo en un epitafio de San Pedro Manrique, en el que la forma Sem(pronie) Titulle es consecuencia de la práctica de simplificar el diptongo (Gómez-Pantoja 1992, 922-923; HEpOl 12888).

2-3. Dada la difícil secuenciación del texto, es necesario tratar conjuntamente ambos renglones. En la lín. 2, lo que queda de la tercera letra consiste en dos trazos rectos divergentes, uno vertical y otro inclinado, por lo que parece lógico reconstruir una $\mathrm{R}$ a la que le falta el bucle superior; la primera cruz representa el astil y las dos líneas rectas perpendiculares características de la parte alta de una $\mathrm{E}$ o F, y la segunda cruz es insegura porque se trata de una forma lanceolada vertical cuyos dos rasgos curvilíneos solo se unen en la parte de arriba, que puede pasar por una A mal hecha o una $\mathrm{R}$ cursiva o una hedera de factura descuidada. Sigue la clara serie AN/O que, aunque parece corresponder a la mención de la edad, está separada del numeral XXI por una rasura de 2 o 3 letras y el grupo SM. En estas condiciones, hay dos posibles lecturas alternativas, ambas con inconvenientes:

A) Sur(--) f(iliae) (hedera) an(n)o(s) [duo]s m(enses) XXI. En esta versión se toma la primera cruz como una F y la segunda como un diacrítico. Sur(---) es un radical presente en nombres propios como Sura, Suris, Surio, Surus y sus derivados, que son corrientes en las inscripciones hispanas y por ello considerados indígenas, aunque resulta difícil distinguirlos de los procedentes de otras tradiciones onomásticas, como sucede, por ejemplo, con los formados a partir del étnico Syrius/Surius (Palomar Lapesa 1957, 99; Albertos Firmat 1966, 215; Vallejo 2016, 165: Siriscum y 417: Surio y Surus) ${ }^{6}$. La expresión de la edad sigue un formulario que apareció en Roma en el tránsito del siglo i al II, difundiéndose también por las provincias; y con él también se extendió el idiotismo de sustituir el ablativo de duración por acusativo ${ }^{7}$. Este modismo se aplicó tanto a niños de corta edad como a adultos y en ambos casos hay ejemplos en los que la cuenta de meses supera los del año.

\footnotetext{
5 Nuestro agradecimiento y aprecio a la propietaria del epígrafe, Elia Lería, por su amabilidad y acogida siempre que hemos acudido a inspeccionar la pieza.

6 En Hispania de las cuarenta y tantas ocurrencias del cognomen Sura, la mitad se deben a las inscripciones que honraron, directa o indirectamente, a L. Licinius Sura (Rodà de Llanza 2014).

7 Vid. Konjetzny 1908, 319-320 para los ejemplos urbanos; en África, Söderström 1924 mostró los casos
}

en los que el uso del ablativo para toda la fórmula predomina sobre los que lo hacen en acusativo y que estos lo hacen sobre los que llevan los ańos en ablativo y los meses y los días en acusativo y que este modismo se hizo común en las inscripciones más modernas. Suárez Martínez 1994 analiza los aspectos sintácticos de la fórmula $\mathrm{y}$, de paso, se refiere a los casos hispanos a partir de los datos de Vives 1971. 
B) Surẹa Ano[ni]s m(ensibus) XXI. Aquí, en cambio, se opta por resolver los signos inseguros como E y A, y mientras la metamorfosis de la primera vocal necesita justificación (Kovács et al. 2009-2011, cat. n. ${ }^{\circ}$ 812: Syrea), ninguna se precisa para Ano, que es un corriente antropónimo hispano.

4. Mientras que de la fórmula sepulcral solo quedan rasgos amorfos, las siglas del admonitum al difunto, aunque de trazo descuidado, se aprecian bien; les sigue un signo indistinto, asimilable al también enigmático de lín. 2 y una $\mathrm{D}$ de significado desconocido que no parece tener nada que ver con el renglón siguiente, porque ni Destitutus, $-a$ ni sus variantes sincopadas están atestiguados como antropónimos.

5. +++TVTAE, lapis: la primera cruz corresponde a un claro signo circular, minuto y secante con la alineación superior del renglón, la segunda cruz representa el ángulo recto de la esquina superior izquierda de una letra, y la tercera, la curva superior de una $S$, un rasgo serpentino. Sin embargo, la secuencia . TVTAE obliga a leer [R]estuta, la frecuente haplología de Restituta.

5-6. $V N C S+T$ es un grupo sin sentido.

6. Mao es un insólito nombre personal, con variantes: Licinius Mao de Aix-en-Provence (Mocci \& Nin 2006, 345), Maodius Veniteriani filius de Dougga (CIL VIII 1521), Maogounus Invictionus de Montier-sur-Saulx (CIL XIII 4668).

7. Puede leerse vota eo, pero es difícil explicar su significado en el contexto del epígrafe.

8. MELIDIAETTITI., lapis: Melidia es un nombre femenino inédito, pero consta su contraparte masculino Meliddius de Luxeuil-les-Bains (CIL XIII 5439, 4, 72). El grupo ETT debió causar problemas al grabador porque después de haber escrito una doble E, corrigió la segunda alargando hacia la derecha su brazo superior y labrando a continuación una $\mathrm{T}$ longa. La forma abreviada de Titi(us) se repite en otros epígrafes: D. Titi. L. l. de Collemancio $(A E$ 2013, 454), Titi. Faustinus de Sremska Mitrovica (Mirković 1994, 374, cat. n. ${ }^{\circ}$ 22), Titi Faustinus bf cos. de Budapest (Kovács et al. 2009-2011, cat. n.o 311).

10. T.SV F, lapis, una insólita secuencia, que aquí se tiene, fue la declaración de autoría del epitafio. La abreviatura t(itulus) no es muy corriente, pero aun así existen más de una docena de ejemplos hispanos de su empleo; por su parte $s u(---)$ aparece en cierto número de inscripciones tardías como compendio del posesivo en sus diversas formas: filia su(a) de Talah (CIL VIII 529), f(ilio) s(uo) de Theveste (ibid. 16626), pius in su(is) de Cantigi (CIL II $\left.{ }^{2} / 7,55\right)$, su(is) sum(p)tibus fecit de Madauros (Gsell 1922, cat. n.o 2525).

11. Lo único claro es el antropónimo y el numeral del final, que puede corresponder a la edad del difunto; los restantes rasgos son indistinguibles o incomprensibles.

La mala calidad del soporte y la impericia del grabador dificultan la lectura y comprensión del epígrafe. Parece tratarse de un epitafio múltiple, en el que solo el nombre de la primera difunta se ajusta al formulario ordinario. Del resto de los individuos mencionados no queda claro si comparten el entierro o son deudos y parientes de la muerta, como tampoco es patente cuál es la relación con todos ellos del último finado.

La onomástica de la difunta se ajusta a lo habitual en la zona, salvo por el añadido de un tercer elemento (o mención de origo) que es inusual en el conjunto. El resto (salvo Titius Maternus) emplean nomina simplicia, lo que suele ser indicio de modernidad, como también lo son las abreviaturas y siglas poco corrientes. 
D. Santa Cecilia 2 (figs. 4 y 5)

Bloque rectangular de arenisca oscura de $52 \times 91 \times 11 \mathrm{~cm}$. A diferencia de la pieza anterior esta tiene pulida la cara inscrita, su estado de conservación es medianamente bueno, salvo por el gran desgaste que afecta al borde superior y que hace sospechar que se usó de umbral o peldańo. Ocupando toda la cara grabada se aprecia el contorno de una tabula ansata levemente hundida y que ha perdido el lado superior por la erosión citada. Esa cartela, cuyas asas están flanqueadas por sendas parejas de hojas lanceoladas, mide $45 \times 60 \mathrm{~cm}$ y contiene seis líneas de texto inciso, escrito con letras capitales de factura rústica y de altura variable; en el primer renglón miden $5,2 \mathrm{~cm}$, en el segundo 12,5 y las de los restantes van disminuyendo su altura hasta los $4,5 \mathrm{~cm}$ del renglón final. Nótese el excesivo uso de abreviaturas, algunas de las cuales no son transparentes, y el irregular empleo de la interpunción para separar sílabas. Mientras que estos rasgos se encuentran en otras inscripciones de la comarca, las proporciones del soporte y su labra hacen de esta lápida un unicum, porque el gusto prevalente se inclinaba más por los monumentos verticales y los característicos bustos y las escenas de animales como motivos decorativos.

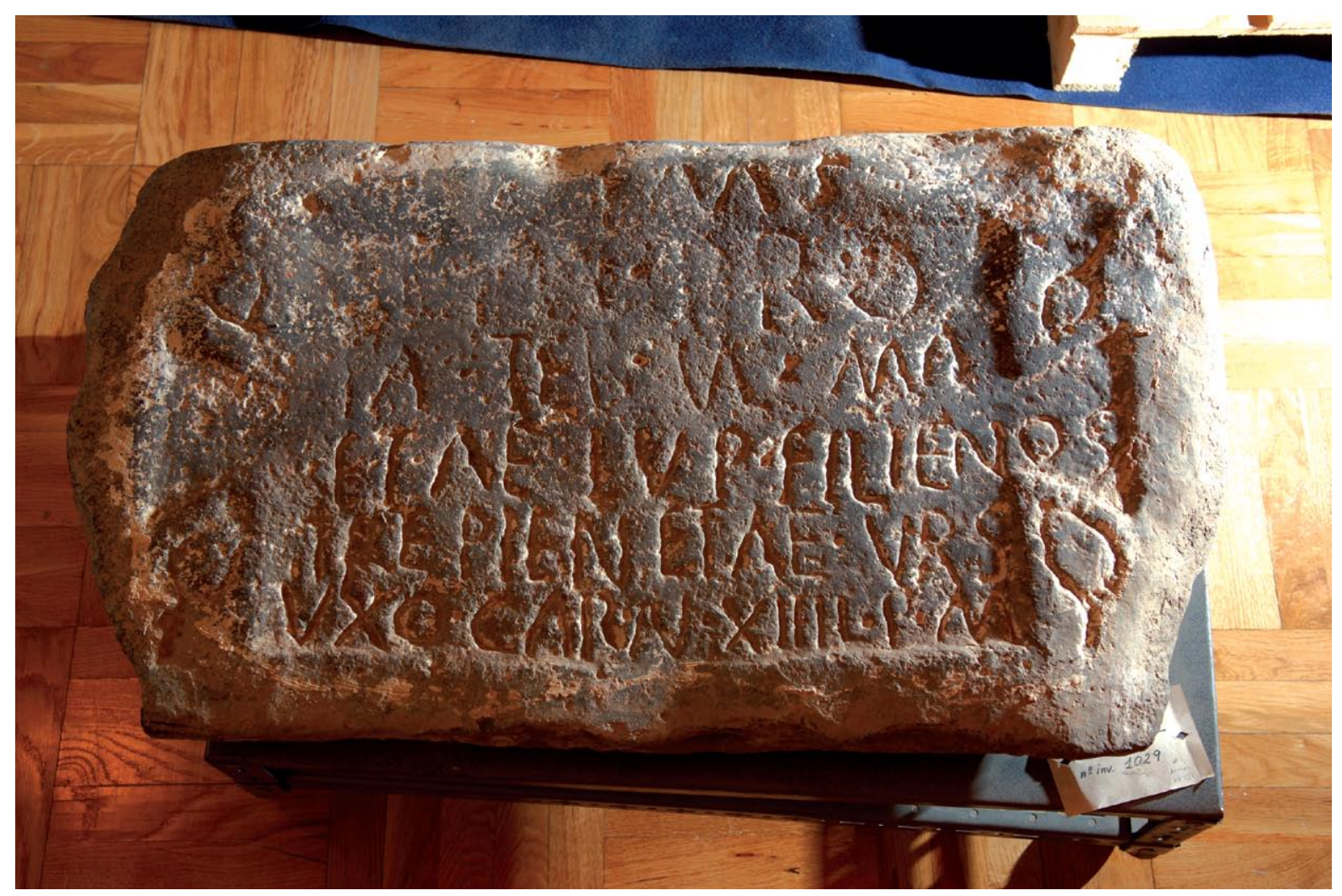

Figura 4. Santa Cecilia 2.

La inscripción es conocida desde principios del xvinI, según consta en el anverso y reverso de una scheda con un imperfecto apógrafo de la misma, conservado en la biblioteca del monasterio de Santo Domingo de Silos, "hallóse... trabajando (al presente de 1737) en la iglesia de Sta. Cecilia, 
de Yanguas junto (5 leguas) de Soria» (Pradales \& Sagredo 1994, 24-25). La noticia pasó desapercibida hasta 1992 y por ello se considera que la editio princeps es la de Ortego, quien la vio y describió a mediados de los años 70 del pasado siglo, cuando estaba empotrada en el muro del atrio lateral de dicha iglesia (Ortego 1976, 257-258; Jimeno 1980, 124; $\mathrm{HEpO} O \mathrm{O} 28818$ ). Vista y fotografiada en 1982 por uno de nosotros (JGP), quien la encontró encalada y difícilmente legible, por esa misma época fue también vista por H. Pascual (Pascual \& Pascual 1984, 82). Cuatro años después de su primera visita, JGP constató que de la inscripción solo quedaba la huella de su alojamiento en la pared. En 2005, sin embargo, supimos de H. Pascual que entre 1982 y 1986 se había extraído el monumento para entregarlo al Museo Numantino, donde está depositada con inv. n. ${ }^{0}$ 92/132 (Alfaro \& Belinchón 2006, 23).

La lectura se hace a partir de los análisis realizados en 2006 y 2011, año este en que estuvo expuesta en Bretún.

$\mathrm{D}$ (is) M(anibus) s(acrum) / Sempro(nius) ${ }^{3} /$ Pater(nus) Val(eriae) ma(tri) / et Ae(miliae) Lup(ianae) filie nos/tre pien(tisimae) et Ae(miliae) Urs(--- $6 /$ uxo(ri) car(issimae) an(norum) XIIII $\mathrm{p}$ (lus) $\mathrm{m}$ (inus).

2. SEM.PRO lapis, [---]SBRO Ortego, Jimeno, Pascual \& Pascual.

3. PA.TER, lapis; pat(er) et: Ortego, Jimeno, Pascual \& Pascual.

4. NOS escrito en el interior del asa derecha, fuera del campo epigráfico.

5. Ae(lia) Urs(ula) edd.

La restitución del texto ha sido disputada. Por un lado, la sintaxis es compleja porque la mención de pater y $m a(t e r)$, conjuntadas por et según los editores anteriores, y seguidos por filia nostra, inducen a pensar que el siguiente nombre corresponde al fruto de esa coyunda. Sin embargo, no se ha dado relevancia al hecho de que pater aparezca con interpunción silábica y compendiado de forma menos radical que el resto; de ahí la propuesta de una nueva restitución, que mejora la inteligencia del epígrafe. El primer nombre de las dos últimas difuntas ha sido unánimemente restituido como Ae(lia), pero ya se ha dicho que la popularidad de Aemilius, - a en la zona lo convierte en la opción más segura. Aplicando el mismo principio a los cognomina de ambas, Lupa y Ursa son mucho más corrientes en el área céltica de Hispania que Lupiana y Ursula, aunque esto queda anulado porque uno y otro están testimoniados en otro epígrafe de la zona, el primero con toda certeza y el otro con probabilidad (Espinosa \& Usero 1988, 480-481; Ortego 1988, 340-341; HEpOl 15196).

Se trata por tanto del epitafio de un peculiar grupo familiar: un viudo, su madre y la hija del primero con una jovencísima esposa que debió morir post partum; la hija parece haber sido criada por el padre y abuela y de ahí que justamente se la designe como filia nostra. El que madre e hija compartan el primer nombre es un hecho banal porque para la época de esta inscripción (siglos III-IV), algunos populares nomina gentilicia, arrastrados por el ejemplo de nombres imperiales como Aur(elius), Fl(avius) y Val(erius), se habían convertido en praenomina funcionales (Salway 1994, 134-140).

EL ORIGEN DE LA ONOMÁSTICA INDÍGENA: UNA LECTURA ARQUEOLÓGICA DE BASE ETNOGRÁFICA

Adelantábamos al inicio las características del corpus epigráfico comarcal compuesto por 5 aras, otras 5 inscripciones avanzadas, dos de las cuales se han tratado aquí (Santa Cecilia 1 y 2), y 29 es- 
telas altoimperiales en las que nos vamos a centrar. Se trata de un conjunto muy uniforme en cuanto a tipología del monumento, decoraciones y fórmulas al que hemos calificado como taller (el taller de Tierras Altas), en el que aparece una particular onomástica con buenos ejemplos en las otras dos piezas que hemos presentado (Villartoso 1 y 2): Belscon, (-)aurce, Bugansonis (gen.), Sulagessia, Udanus.
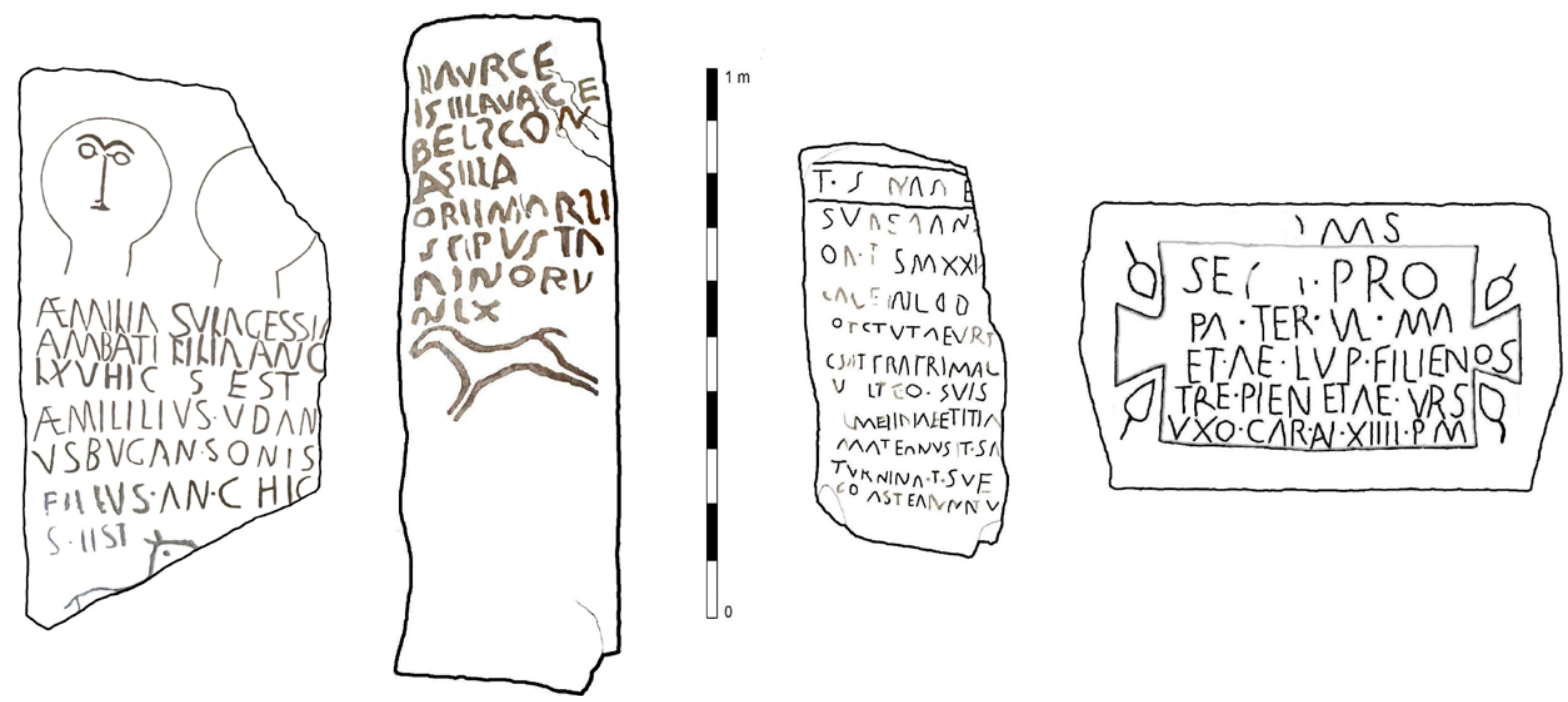

Figura 5. Villartoso 1 y 2, Santa Cecilia 1 y 2.

Adelantábamos también las características geográficas del territorio. Para contextualizar y analizar la presencia de esta onomástica no indoeuropea, desde un punto de vista arqueológico, es importante insistir en su medio físico, montañoso y elevado, lo que condiciona de forma determinante su economía y sus relaciones con el exterior, como bien queda plasmado en sus formas de vida tradicionales, en su etnografía.

Alcanzan los valles de Tierras Altas alturas notables, desde algo más de $1.000 \mathrm{~m}$ en el fondo de valle, donde se emplazan sus dos pequeños núcleos urbanos altoimperiales, Los Casares de San Pedro Manrique y Las Gimenas de Villar del Río, hasta los 1.700-1.600 m que mantiene la línea del interfluvio Duero-Ebro entre los puertos de Piqueras y Oncala (Montes Claros y Sierra de Alba). Es esta vertiente septentrional del interfluvio, la del Ebro, en la que se concentran dos tercios de las estelas altoimperiales (19 de las 29 piezas), con la mayoría de la onomástica no indoeuropea (12 de los 14 nombres) y los nomina latinos más repetidos en la comarca, las familias Antestia, Aemilia, Valeria y Sempronia, que parecen apuntar a vínculos con el Ebro en general y en especial con $C a-$ lagurris. Las aldeas actuales de esta zona situada al pie del interfluvio y los vici altoimperiales localizados en ella rondan, e incluso sobrepasan, los 1.200-1.300 m de altura. Sus respectivos territorios de explotación y especialmente sus espacios adehesados, ascienden las laderas hasta alcanzar en 2-4 km el cambio de cuenca con el Duero, terreno de pastos que pasan por ser los más finos de la Sierra. Una decena de kilómetros aguas abajo de Cidacos y Linares se ha abierto algo el paisaje, lo que permite un mayor aprovechamiento agrícola dentro de la general predisposición ganadera del territorio; aquí se localizan los poblados centrales, tanto los altoimperiales como las villas moder- 
nas, de cuyo entorno proceden 6 estelas. Con el fluir de los ríos, pasadas las villas, el eco del taller de Tierras Altas se desvanece hasta desaparecer al poco de entrar en la sierra riojana, donde se localizaron las 4 piezas restantes.

En definitiva, con la dispersión de epígrafes que contamos en la actualidad y con la información que de ellos se desprende, parece que el área donde se concentra el trabajo del taller está en el sector más alto y ganadero de Tierras Altas, el nacimiento del Cidacos con su afluente Baos, y del Linares, perdiéndose progresivamente su presencia con el fluir descendente de sus aguas: muy mermada en torno a los dos pequeños núcleos urbanos, y desapareciendo su forma de hacer con el final de la comarca natural e histórica de las viejas comunidades de villa y tierra de Yanguas y San Pedro Manrique (fig. 6).

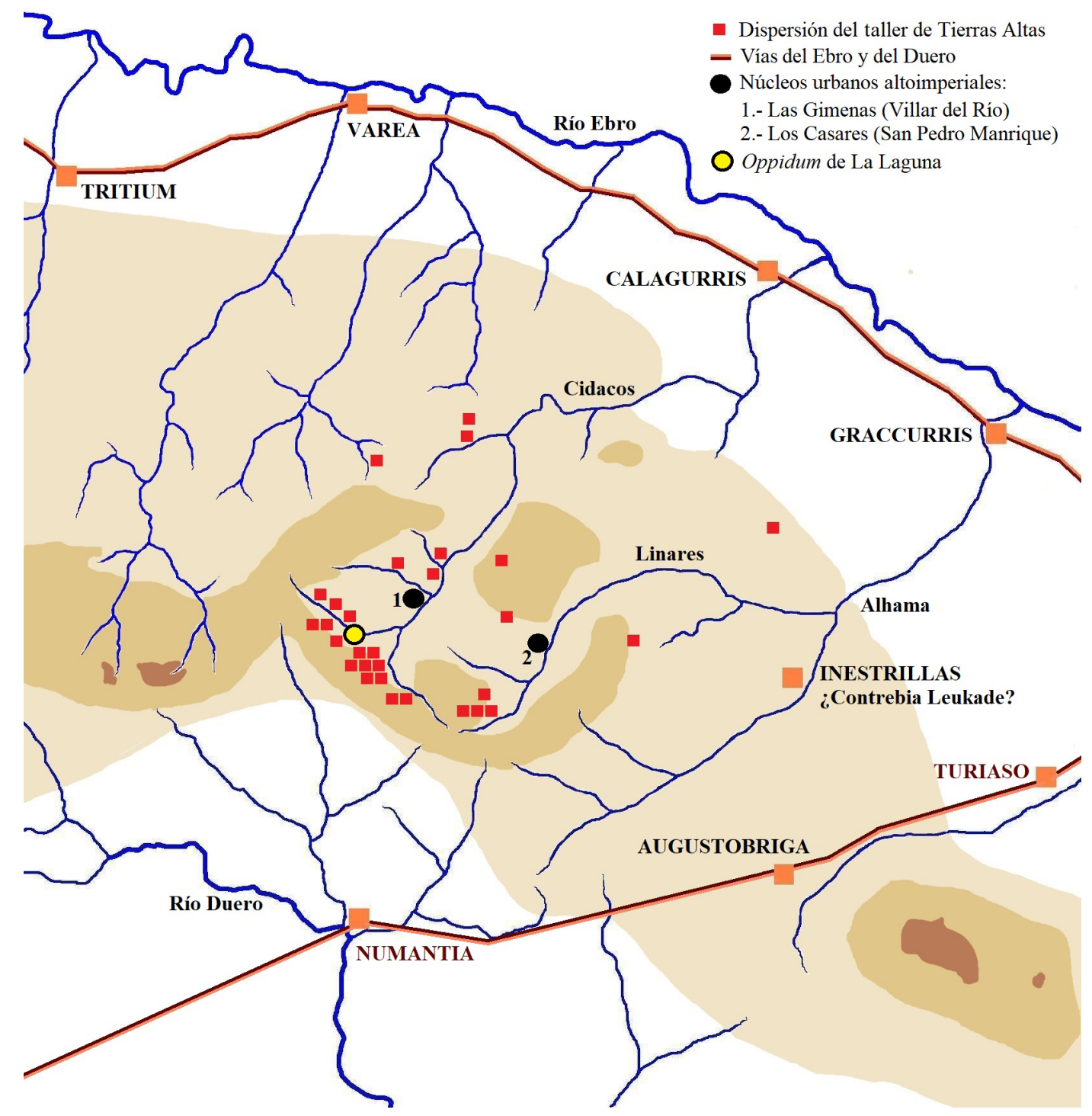

Figura 6. Dispersión del taller de Tierras Altas y contexto poblacional. 
Resulta imprescindible ya incidir en la riqueza por antonomasia de Tierras Altas, un territorio profundamente ganadero y trashumante con óptimos pastos de verano, los estivaderos, capaces de alimentar decenas de miles de cabezas de ganado, una de las formas de riqueza en la Antigüedad, cuyo mantenimiento de octubre a mayo hay que buscarlo lejos de la nieve y el hielo que cubre la Sierra. La amplia cabaña necesita pastos de invierno, invernaderos que durante el último milenio se han localizado al sur de Castilla-La Mancha y Extremadura, y a los que se llegaba tras unos 30 días de recorrido por las vías pecuarias, cañadas de las que nuestro territorio es raíz de una de ellas, la Cañada Real Soriana Oriental (Río 1857). Esta alternancia anual, la trashumancia, supone el desplazamiento durante la mitad del año de una parte pequeña de la comunidad, pastores en su plenitud, al mismo tiempo que se queda en la Sierra - el territorio que sienten y les es propio- el grueso de su población.

En el territorio de los antiguos vici que explotaron los pastos de las laderas norte de Montes Claros y Alba aparecen nombres no indoeuropeos de niñas, mujeres, ancianos y varones de todas las edades. Este dato deja claro que, hace dos mil años, la base del grupo humano del que ha trascendido esta particular onomástica indígena, estaba en los altos valles de Cidacos y Linares, no en sus invernaderos, en el muy probable supuesto de que durante el cambio de Era hubiese ya movimiento cíclico anual con el ganado. El potencial económico, es decir, su propia subsistencia, así como sus evidentes limitaciones invernales obligan a pensar que, como en los últimos siglos, serían algunos de sus pastores los que se desplazarían a los invernaderos con el grueso del ganado.

La vida tradicional en la Serranía ha estado condicionada por la necesidad subsistencial del movimiento anual, lo que obliga a mantener relaciones de convivencia estables con territorios ajenos y más o menos alejados, los que atravesaban sus rutas ganaderas y sobre todo con el marco de sus invernaderos. Por otro lado, está el natural aislamiento de estas zonas montañosas y elevadas, a desmano de las rutas de comunicación, con un clima severo por sus fríos y largos inviernos frente a un verano corto y suave, valles cuyas alturas son fronteras naturales y en cuyo interior barrancos y torrenteras constituyen un pequeño e inquietante laberinto para toda persona ajena al territorio. En definitiva, una comarca natural con unas características físicas propicias a generar un grupo humano cerrado, tendente a organizarse en una misma unidad social y administrativa envuelta en sí misma, circunstancia muy matizada por su potencial económico necesitado de mantener unas relaciones estables con gentes alejadas de sus montañas, como mínimo las que controlaban el territorio de sus invernaderos.

La economía trashumante tradicional se ha sustentado en el ganado ovino, miles de cabezas de merinas, complementada con una agricultura cerealista base, más un número indeterminado de ganado vacuno, siempre minoritario. Los restos faunísticos localizados en los dos oppida comarcales de los siglos III-I a.C., El Castillo de La Laguna en el Cidacos y Los Casares de San Pedro en el Linares, apuntan a una mayor incidencia del bovino en esos siglos previos a la estabilidad proporcionada por Roma que en la economía tradicional moderna. Son estudios muy someros limitados al análisis estadístico de depósitos asociados arqueológicamente a vertederos domésticos que, generalmente, apuntan más al peso de cada cabaña en la dieta que a la contabilización fiel de cada especie; en cualquier caso, los datos no dejan de ser el reflejo de una determinada realidad (Alfaro 2018b, 260-262). Grosso modo el número de restos de ovicaprinos dobla al de bóvidos que, sumados, vienen a suponer entre un $85-90 \%$ de los restos de fauna localizados en estos basureros. La importancia sicológica del vacuno en el universo simbólico de las gentes de las estelas se aprecia, además, en los propios monumentos funerarios que tratamos, al ser el animal más representado, casi exclusivo en los varones si exceptuamos al caballo, y presente en las féminas donde, junto con la cierva amamantando, trascienden al animal en sí para convertirse en símbolos para la eternidad del difunto (Alfaro \& Belinchón 2006, 10-13; Alfaro 2014, 325-326). 


\section{El origen de la onomástica no indoeuropea, ¿continuidad o ruptura?}

La presencia de nombres no indoeuropeos en torno a los manantiales de estos afluentes de la margen derecha del Ebro, cuestiona el mapa étnico-lingüístico tradicional que sitúa en estos valles en los siglos previos a la entrada de Roma a gentes célticas, los pelendones, integrados en el orbe celtibérico. También, y en cierto modo, choca con la cultura material de los dos oppida comarcales que vivieron las guerras de conquista y el conflicto sertoriano, plenamente celtibérica y numantina. Decimos «en cierto modo", porque la cultura material celtibérica se extiende en este momento también por toda la ribera riojano-navarra del Ebro, con ciudades como Kalakorikos/ Calagurris, celtibérica durante la conquista y que en Ptolomeo (II, 6, 67) aparece como vascona. Es por ello que una de las preguntas clave concierne al origen de esta onomástica comarcal, siendo dos las posibilidades básicas: una, que estas gentes estuviesen asentadas en Tierras Altas desde tiempos ancestrales, quizás desde que hay evidencias de un poblamiento estable, fijado al territorio, lo que supone remontar su origen a un Bronce Final o el incipiente Primer Hierro con sus poblados fortificados de la cultura Castreña; otra, que haya habido un desplazamiento del grupo humano con esta onomástica en un momento anterior al de las estelas. En este caso es lógico pensar que el movimiento hubiese sobrevenido como consecuencia de los conflictos en los que se ve envuelto el territorio, previos al cambio de Era, las guerras de conquista y sertoriana.

\section{Reflexiones desde la continuidad}

Descritas las características físicas de Tierras Altas, un territorio de alta montaña, con una economía subsistencial exigente, un clima difícil por sus crudos y largos inviernos, sin especial relevancia estratégica en la red de comunicaciones, de acceso y movilidad interna complicados para toda persona ajena a él, tendente además, y en consecuencia de lo anterior, a generar una sociedad cerrada y envuelta en sí misma..., descrita esta visión del territorio como decimos, una de las lecturas más plausibles sería asumir una raigambre ancestral de este grupo humano en el territorio. Estaríamos ante unas gentes hechas a la austeridad de la Sierra, sin atractivo vital ni económico para ambiciones ajenas si exceptuamos sus pastos estivales. Esta hipótesis llevaría a remontar su origen hasta la evidencia de los primeros poblados estables en la zona, los últimos compases de la Edad del Bronce y el Primer Hierro. La población itinerante, nómada o seminómada, que aprovechaba regularmente estos pastos serranos en tiempos protohistóricos acabaría por hacer suyas las hierbas estivales fijando a todos o a una parte de su excedente demográfico en poblados estables, emparentados por tanto con el grupo original que, en su itinerancia, contaba con los pastos de invierno. La lógica geográfica y económica apunta a la cuenca baja de sus propios valles del Ebro, la actual ribera riojano-navarra y todo su entorno, a dos jornadas a pie de nuestras cumbres. Movimiento primigenio entre el fondo de valle y las zonas altas que justificaría la génesis lingüística de la onomástica en cuestión.

\section{La posibilidad de una ruptura}

La pujanza política y cultural del mundo celtibérico durante el Segundo Hierro en el oriente de la Meseta iría calando en la Sierra y en la ribera del Ebro. Roma irrumpe en su curso medio desde las primeras décadas del siglo II a.C., y se asienta en torno a la confluencia con el Ebro de uno de nuestros valles fundando Gracurris. Los montańosos cursos altos de Cidacos y Linares se convierten así en obligado parapeto del mundo indígena, liderado en la Celtiberia Ulterior por los aréva- 
cos del oriente meseteño. Sería en este momento cuando las gentes del interior de nuestra Serranía, sus dos oppida, se verían impelidos a coaligarse con las ciudades celtibéricas del entorno ante el interés común. Para Roma serían dos ciudades celtibéricas más, lo fuesen o no desde el punto de vista étnico-lingüístico, pero sí integradas en las grandes y genéricas coaliciones celtibéricas a las que se enfrenta (Sánchez Moreno et al. 2015, 77). Desde los conflictos de conquista hasta la derrota de Sertorio, todo este sector del valle del Ebro se convierte en un territorio de frontera en el que se suceden las crisis bélicas con momentos de estabilidad, se fundan ciudades y se arrasan otras, hay movimientos de gentes, a veces voluntarios, otras forzosos. Este sería uno de los escenarios más probables para contextualizar una hipotética ruptura étnico lingüística en los cursos altos de Cidacos y Linares. Se apunta en las fuentes que el desplazamiento de determinados grupos humanos fue una herramienta utilizada por Roma, tanto para reprimir como para premiar desafecciones y afecciones a su causa. Un ejemplo cercano lo aporta san Isidoro (Etym. IX, 2, 107) que cuenta cómo Pompeyo, tras acabar con Sertorio y sus seguidores, parece que pobló con vascones un sector de Aquitania (Gómez-Pantoja 2007, 342-343; Vidal 2012, 129-130).

Un hecho que podría apuntar en esta dirección es la destrucción violenta del oppidum del valle del Cidacos, El Castillo de La Laguna, que se arrasa. Emplazado en el sector más alto del valle, en un altozano elevado sobre los barrancos del Baos, afluente del Cidacos, a sus $1.273 \mathrm{~m}$ domina directamente los pastos de Montes Claros y Alba. Un paseo por las ruinas de sus defensas delata embates en varios puntos en los que aún ruedan abundantes adobes hipercalcinados, y en el interior del poblado son numerosas las evidencias de incendio (Alfaro 2018b, 303-307). El hecho es que este oppidum no sobrevive a los conflictos y que en su entorno, a un kilómetro en vuelo, vivieron varias generaciones después (-)Aurce, Belscon, Bugansonis (gen.), Sulagessia... y el ya emblemático Sesenco, este en un vicus situado a no más de $300 \mathrm{~m}$ de las ruinas de El Castillo. La mayoría de los nombres indígenas que nos ocupan se localizan en el espacio que rodea al viejo oppidum, en el que se dispersan pequeñas aldeas altoimperiales en las que tuvieron que habitar: La Sancha (La Laguna), Los Corralejos/Entre los Prados (Villartoso), El Collarazo (Santa Cecilia) y La Muela (Valloria) (Alfaro 2005, 310-317). El hipotético vacío poblacional que había dejado El Castillo de La Laguna pudo haber sido aprovechado por gentes de vocación ganadera, interesadas en estos pastos estivales en cuyo territorio se asentarán, haciéndolo propio y cuyo eco nos ha llegado a través de su onomástica. Resulta chocante comprobar cómo en Villar del Río, localizado al pie de Las Gimenas, el pequeño núcleo urbano altoimerial del Cidacos, no se conoce ninguna estela del taller, cuando la lógica epigráfica llevaría a concentrar aquí una parte significativa de los testimonios. Siempre y cuando nuevos hallazgos no rompan esta inercia, se trata de un dato más que inclina al taller de Tierras Altas hacia el lado de un contexto ganadero y rural, usado este último término como contraposición a los dos pequeños núcleos urbanos comarcales, Las Gimenas y Los Casares.

El hecho, ya indudable, es que la tradicional filiación céltica de este sector soriano del valle del Ebro no es indiscutible, ni mucho menos. Tampoco su radical negación por la constatación de algunos nombres y teónimos. Que de las estelas se infiere cierto mestizaje onomástico, con predominio más que claro entre los indígenas de los no indoeuropeos es evidente, dentro de una mayoría general de los latinos. En definitiva, solo nos queda subrayar la complejidad étnico lingüística de la margen derecha del Ebro en su transición alto-medio, la actual ribera riojano-navarra y sus montañas meridionales hasta alcanzar la actual provincia de Soria, un territorio donde parece coexistieron y convivieron un conglomerado de pueblos y lenguas — celtibérica, ibérica y vascona—, situación que se iría homogeneizando con el afianzamiento del latín. 


\section{BibliografíA}

Albertini, E.,1923, Les divisions administratives de l'Espagne romaine, Paris: E. de Boccard.

Albertos Firmat, M. a L., 1966, La Onomástica Personal Primitiva de Hispania Tarraconense y Bética, Salamanca: Instituto «Antonio de Nebrija».

Alfaro Peña, E., 2005, Castillejos y Villares. Modelos de poblamiento antiguo en el interior del Sistema Ibérico, Soria: Soria Edita.

Alfaro Peña, E., 2008, «Reliquias celtibéricas en Tierras Altas: dos fíbulas zoomorfas y un fragmento ancoriforme», Revista de Soria 61, 27-42.

Alfaro Peña, E., 2014, «Iconografía Funeraria Indígena. Reflejos, lecturas y pautas en estelas de Tierras Altas, Soria», en: F. Burillo, M. Chordá (eds.), VII Simposio sobre los celtíberos. Nuevos hallazgos, nuevas interpretaciones, Teruel: Fundación Segeda-Centro de Estudios Celtibéricos, 321-330.

Alfaro Peña, E., 2018a, «Los Casares de San Pedro Manrique: un viejo oppidum, una pequeña ciudad», en S. Martínez, J. Santos, L. J. Municio (eds.), El Urbanismo de las ciudades Romanas del Valle del Duero, Segovia: Museo de Segovia, 51-69.

Alfaro Peña, E., 2018b, Oppida y etnicidad en los confines septentrionales de la Celtiberia, Tesis Doctoral de la Universidad de Valladolid.

Alfaro Peña, E., \& G. Belinchón, 2006, Costumbres romanas para la muerte en Tierras Altas de Soria, León.

Caro Baroja, J., 1946, Los Pueblos de España, Barcelona: Istmo.

Delamarre, X., 2007, Nomina Celtica Antiqua Selecta Inscriptionum. Noms de personnes celtiques dans l'épigraphie classique, Paris: Errance.

Espinosa Ruiz, U., 1992, «Los castros soriano-riojanos del Sistema Ibérico: nuevas perspectivas», en: C. de la Casa (dir.), II Symposium de Arqueología Soriana. Actas, Volumen II, Soria: Diputación Provincial, 899-913.

Espinosa Ruiz, U., \& L. M. Usero, 1988, «Eine Hirtenkultur im Umbruch. Untersuchungen zu einer Gruppe von Inschriften aus dem conventus Caesaraugustanus (Hispania Citerior)», Chiron 18, 477504.

Gómez-Pantoja, J., 1992, «Nuevos testimonios epigráficos sorianos», en C. de la Casa (dir.), II Symposium de Arqueología Soriana. Actas, Volumen II, Soria: Diputación Provincial, 918-925.

Gómez-Pantoja, J., 2007, «Una tierra de emigrantes», en: M. Navarro, J. J. Palao (eds.), Villes et Territoires dans le Bassin du Douro à l'Époque Romaine, Bourdeaux: Ausonius, 341-353.

Gómez-Pantoja, J., \& E. Alfaro Peña, 2001, «Indigenismo y Romanización en las Tierras Altas de Soria. Nuevos testimonios epigráficos», en: F. Villar, M. a P. Fernández (eds.), Religión, Lengua y Cultura Prerromanas de Hispania, Salamanca: Universidad, 169-187.

Gorrochategui, J., 1984, Onomástica Indígena de Aquitania, Bilbao.

Gorrochategui, J., 2009, «Vasco antiguo: algunas cuestiones de geografía e historia lingüísticas», Acta Palaeohispanica X, 629-634.

Gsell, S., 1922, Inscriptions latines de l'Algèrie, I: Inscriptions de la Proconsulaire, Paris.

Gsell, S., \& H. G. Pflaum, 1976, Inscriptions latines de l'Algèrie, II.2: Entre Cirta et Thibilis, Thibilis. De Civitas Nattabutum à Tigisis, Région au Sud de Gadiaufala, Thigisis et Sigus, Sifus et environs de Sigus, Sila et environs de Sila, Alger.

Jimeno Martínez, A., 1980, Epigrafía Romana de la Provincia de Soria, Soria: Diputación Provincial.

Konjetzny, G., 1908, «De idiotismis syntacticis in titulis latinis urbanis (C.I.L. Vol. VI.) conspicuis», $A r-$ chiv für lateinische Lexikographie und Grammatik, 297-351.

Kovács, P. et al., 2009-2011, Tituli Aquincenses, vol. 1: Tituli operum publicorum et honorari et sacri (2009). - 2: Tituli sepulcrales et elii Budapestini reperti (2010) - 3: Tituli instrumenti domestici (2011), Budapest.

Mirković, M., 1994, «Beneficiarii consularis in Sirmium», Chiron 24, 345-404. 
Mocci, F., \& N. Nin, 2006, Aix-en-Provence, Pays d'Aix, Val de Durance, Carte Archéologique de la Gaule, vol. 13/4, Paris.

Ortego Frías, T., 1976, «Hallazgos notables de estelas y miliarios hispano-romanos». Celtiberia 52, 251 260.

Ortego FríAs, T., 1988, «Estelas funerarias inéditas, con representaciones bovinas, en territorio arévacopelendón", Gerión [Anejos, I], 324-342.

Palomar Lapesa, M., 1957, La onomástica personal pre-latina de la antigua lusitania. Estudio lingüistico, Salamanca: Instituto Antonio de Nebrija.

Pascual Mayoral, P., \& H. Pascual González, 1984, Carta Arqueológica de La Rioja I. El Cidacos, Calahorra: Amigos de la Historia de Calahorra.

Pradales Ciprés, D., \& L. Sagredo San Eustaquio, 1994, «Estudio onomástico y epigráfico de época romana basado en manuscritos del siglo xviII", Hispania Antiqua 18, 381-432.

Río, C. DEL, 1857, Descripción de la Cañada Soriana, desde Yanguas al valle de La Alcudia, Valencia: Librerías París-Valencia, facsímil de 1995.

RodÀ de Llanza, I., 2014, "Lucius Licinius Sura, Hispanus», en: I. Piso (ed.), Trajan und seine Städte), 21-35.

Salway, B., 1994, «What's in a name? A survey of Roman onomastic practice from 700 B.C. to A.D. 700», Journal of Roman Studies 84, 124-145.

Sánchez Moreno, E., A. Pérez Rubio \& E. García Riaza, 2015, «Fronteras y agregaciones políticas en Celtiberia: datos para un debate», Cuadernos de Prehistoria y Arqueología de la Universidad Autónoma de Madrid, vol. 41, 69-85.

SöDERström, G., 1924, Epigraphica latina africana: de titulis sepulcralibus prosa oratione compositis provinciarum Byzacenae et proconsularis quaestiones selectae, Tesis de Doctorado de la Uppsala Universitet.

SuÁrez Martínez, P. M., 1994, «Vixit annis XXX Menses III dies XIX», en: J. Herman (ed.), Linguistic Studies on Latin: Selected Papers from the 6th International Colloquium on Latin Linguistics, 55-63.

Taracena Aguirre, B., 1933, «Tribus celtibéricas. Pelendones», Homenagem a Martins Sarmento, Guimaraes: Sociedade Martins Sarmento, 393-401.

Vallejo, J. M., 2016, Onomástica paleohispánica. I, Antroponimia y teonimia. I. Testimonios epigráficos latinos, celtibéricos y lusitanos y referencias literarias, Banco de datos Hesperia de lenguas paleohispánicas, vol. III, Vitoria.

Vidal Joan, C., 2012, «El aquitano como lengua céltica (o vascones en Aquitania)», Nouvelle Revue d'Onomastique 54, 129-175.

Vives, J., 1971, Inscripciones Latinas de la España Romana, Barcelona: Universidad de Barcelona. 